\title{
A Quiescent Colossal Radicular Cyst: A Diverse Histological Presentation
}

\author{
V Reshma, Radhika M Bavle, BK Varsha, BM Kavya
}

\begin{abstract}
Radicular cysts are the most frequent inflammatory cysts comprising of about 52 to $68 \%$ of all the jaw cysts. It arises from the epithelial residues in the periodontal ligament space following pulpal necrosis. The distinguished lining of the cystic lumen is hyperplastic stratified squamous nonkeratinizing epithelium showing arcading pattern with dense inflammatory cell infiltrate, cholesterol clefts, Russell bodies and Ruston bodies in the connective tissue capsule. Here, we present an unusual large radicular cyst lined almost entirely by quiescent atrophic epithelium, which is innocuous, persistent, has enlarged overtime, with minimal inflammatory cells in the connective tissue capsule.
\end{abstract}

Keywords: Bilateral lytic lesions, Large radicular cyst, Cholesterol crystals, Quiescent epithelium.

How to cite this article: Reshma V, Bavle RM, Varsha BK, Kavya BM. A Quiescent Colossal Radicular Cyst: A Diverse Histological Presentation. World J Dent 2013;4(4):286-290.

Source of support: Nil

Conflict of interest: None declared

\section{INTRODUCTION}

Radicular cysts are common inflammatory odontogenic cysts comprising of about 52 to $68 \%$ of all cysts of the jaws. They arise in the periodontal ligament (PDL) space from the epithelial residues following pulpal necrosis. ${ }^{1,2}$ The epithelial source is by and large, cell rests of Malassez; other sources such as crevicular epithelium, sinus lining or epithelial lining of fistulous tract, may also contribute. ${ }^{3}$

Radicular cyst generally involves the maxillary anterior teeth at about third to fifth decades of life with a male predominance. The main feature in the diagnosis of a radicular cyst is the presence of a tooth with nonvital pulp and sometimes a sinus opening that may lead from the cyst cavity to the external mucosal surface. ${ }^{1,2}$

Histopathologically, the cyst consists of a cavity lined partly or wholly by stratified squamous nonkeratinizing epithelium which is about 6 to 20 cell thick and may exhibit spongiosis and exocytosis. The variations seen in epithelial lining include keratinization (commonly orthokeratinization), presence of columnar ciliated epithelium or mucosecretory cells. Rushton's hyaline bodies which have a glassy pink appearance of varying shapes are seen in about 2.6 to $10.3 \%$ of the reported cases within the epithelial lining. ${ }^{2,4}$

The fibrous capsule consists of a loose connective tissue adjacent to epithelial lining followed by condensed parallel bundles of collagen fibers arranged peripherally. The wall is inflamed consisting predominantly of chronic inflammatory cell infiltrate. Multinucleated giant cells, neutrophils and Russell bodies can also be seen. Slow accumulation and deposition of cholesterol crystals during the inflammatory process leads to the formation of clefts as these crystals are dissolved out during histological processing. The cholesterol crystals move in the direction of the epithelium-lined cyst cavity since the outer collagenous capsule of the lesion is too tough for the crystals to move through and are thus expelled into cystic lumen. The nature of the cyst lining depends on the age or stage of development of the cyst, or on the intensity of the inflammation. In early cysts, the epithelial lining may be proliferative and show arcading with an intense associated inflammatory process but as the cyst enlarges the lining becomes quiescent and fairly regular with a certain degree of differentiation to resemble an atrophic simple stratified squamous epithelium with minimal inflammation in the connective tissue wall. ${ }^{2,4-6}$

Here, we present a case of bilateral unilocular cystic lesions, one of which was found to be a large radicular cyst with atrophic, quiescent epithelial lining rimming almost the entire cyst wall.

\section{CASE REPORT}

A female patient aged 45 years, reported with a chief complaint of pain and swelling in right cheek region since 9 months. The patient gave a history of the swelling since 10 years associated with intraoral pus discharge. Her medical history revealed her to be hypertensive since 5 years which is controlled by medication. Patient did not have any relevant previous dental history.

On extraoral examination (Fig. 1A), a diffuse swelling of about $4 \times 5 \mathrm{~cm}$ was seen on the right malar prominence extending superioinferiorly from the infraorbital rim to about $1 \mathrm{~cm}$ above the line drawn from the corner of mouth to tragus, and anterioposteriorly from ala of the nose to the midpoint of the ala tragus line. Skin over the swelling was normal but stretched. On palpation, the swelling was firm and tender. The surface temperature was normal with no altered sensation.

A single right submandibular lymph node was found to be enlarged, mobile and tender. It measured approximately about $1 \times 1 \mathrm{~cm}$ in size.

Intraoral examination (Fig. 1B) revealed two soft tissue swellings one each on right and left maxillary posterior region. 


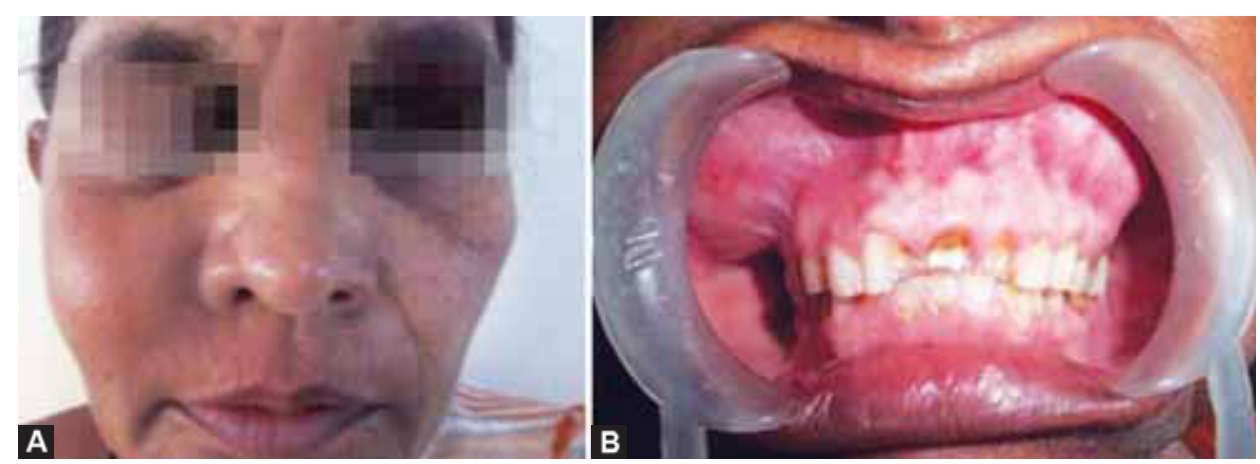

Figs 1A and B: (A) Extraoral diffuse swelling seen on the right malar prominence, (B) intraorally, bilateral swellings seen obliterating the maxillary vestibule

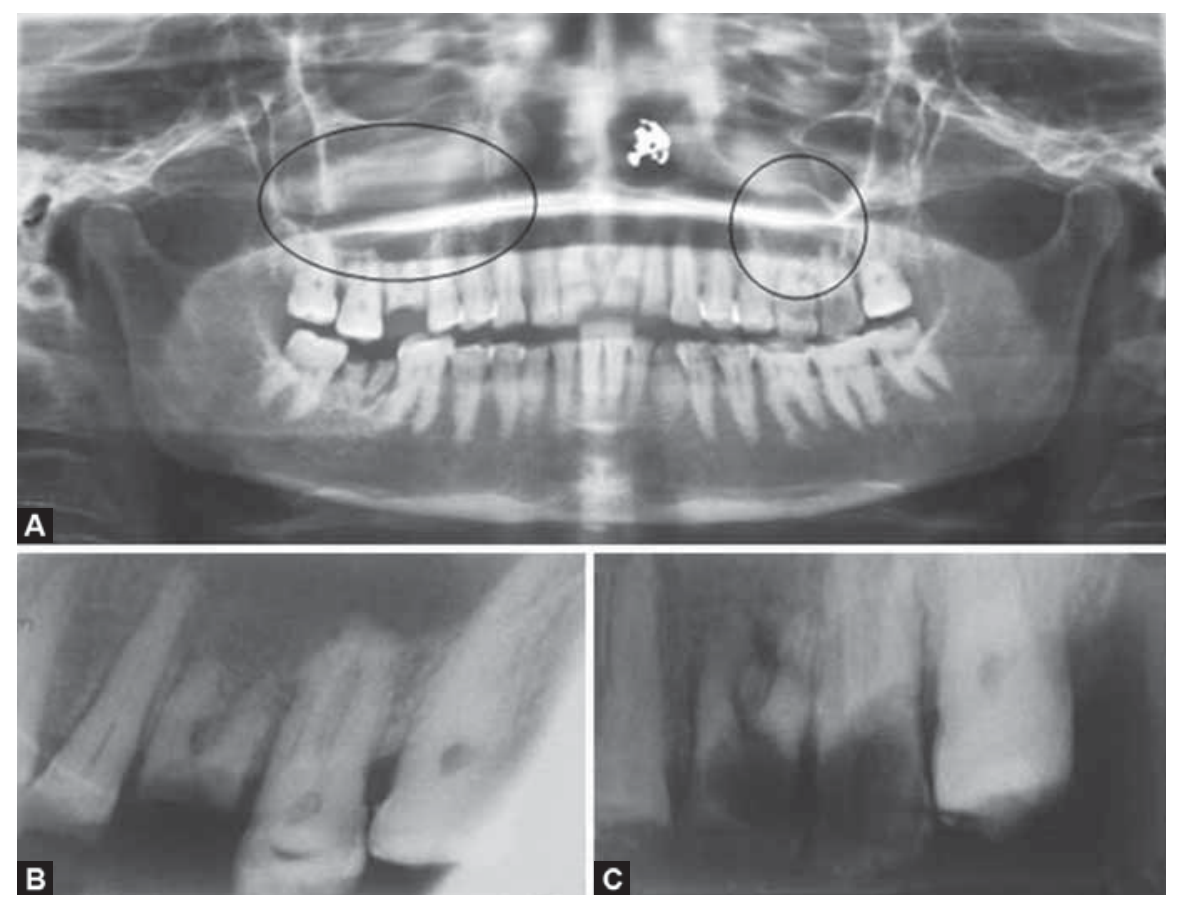

Figs 2A to C: (A) OPG showing bilateral unilocular radiolucencies involving the maxilla over the roots of 15,16 and 17 on the right side and 26 and 27 on the left side, (B) right IOPA showing root stumps of 16 and hazy radiolucency over the root stumps, (C) left IOPA showing grossly decayed 26 and 27

On the right side, the swelling was about $4 \times 1 \mathrm{~cm}$ in size and extended from the canine to third molar obliterating the vestibule. The mucosa over the swelling appeared normal. Root stumps of a carious first molar were present. A sinus opening was observed on the attached gingiva in relation to (irt) first premolar. The swelling was soft to firm in consistency with slight tenderness.

The swelling on the left side was diffuse measuring approximately $3 \times 1 \mathrm{~cm}$, obliterating buccal vestibule and extended from the first premolar to second molar. The mucosa over this swelling appeared normal with a draining sinus present on attached gingiva irt 26. The swelling was soft and tender. Grossly decayed first and second molars were present. The patient had poor oral hygiene with teeth showing generalized attrition.
Orthopantomogram (Fig. 2A) showed full complement of maxillary and mandibular teeth. On the right side a well defined radiolucency involving the apices of 15, 16 and 17 which was merging and involving the floor of the maxillary sinus was seen. On the left side, a well demarcated radiolucency involving the apices of 25, 26 and 27 was present. Root stumps irt 47 was also noted.

Intraoral periapical (IOPA) radiographs (Fig. 2B) taken on right side showed root stumps with furcation involvement and PDL widening irt 16 with hazy radiolucency involving the apices of 15,16 and 17.

IOPA on the left side (Fig. 2C) showed grossly decayed 26 and 27 with furcation involvement and PDL widening irt 26.

As the lesion was bilateral, a cone beam computed tomography (CBCT) was done to find the exact extent and 
nature of the lesion. CBCT (Fig. 3) in coronal view showed a thin corticated unilocular radiolucent lesion measuring about $5 \times 3 \mathrm{~cm}$ in right maxillary posterior region causing buccal cortical plate expansion and involving 15 and 16. A 3D reconstruction image of the right sided lesion (Fig. 4) showed that zygomatic process was not involved.

The swelling on the left maxilla was radiolucent with thin corticated margin with perforation of the labial cortical plate. The swelling measured about $2 \times 2.4 \mathrm{~cm}$ and extended close to floor of left maxillary sinus.

Differential diagnoses considered were radicular cyst and lateral periodontal cyst. Since the lesion was bilateral, differential diagnosis also included infected odontogenic keratocyst, central giant cell granulomas, ossifying fibroma and infected dentigerous cyst.

Aspiration (Fig. 5A) of the right swelling yielded a straw-colored fluid with dense collection of shimmering yellow crystals. Aspiration of the swelling on the left side gave a blood tinged fluid. Smear made of straw-colored fluid showed numerous cholesterol crystals (Fig. 5B) pointing that the lesion was infected and cystic in nature.

The lesion on the right side was excised along with root stumps of 16. Macroscopic examination (Fig. 6) revealed the specimen to be a cystic lesion roughly oval in shape measuring approximately $5 \times 3 \mathrm{~cm}$, creamish brown in color with an irregular outer surface. Cut surface showed a smooth creamish white inner lining with few areas which were close to the apex of the tooth showing yellowish nodules. The cyst wall was firm in consistency.

Histopathological examination revealed a continuous, uniform smooth atrophied stratified squamous nonkeratinizing epithelium of about 2 to 4 layers thick lining almost the entire cystic wall. The lesional tissue which was close to the maxillary sinus showed a normal pseudostratified ciliated columnar epithelium. The sections taken from the yellowish nodules showed hyperplastic stratified squamous epithelium of about 16 to 20 layer thick with an arcading pattern and

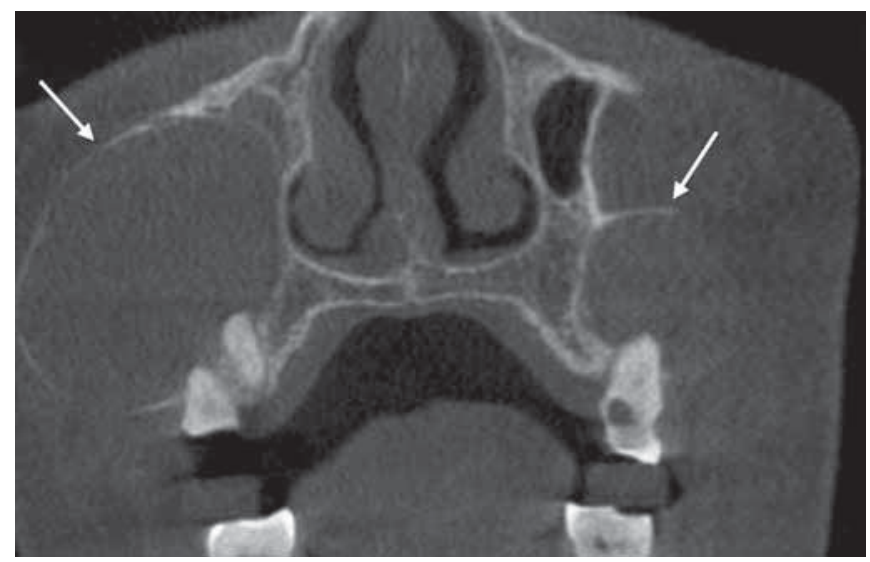

Fig. 3: CBCT showing thin corticated unilocular radiolucent lesion causing right maxillary buccal plate expansion and unilocular left radiolucency with the cortical margin

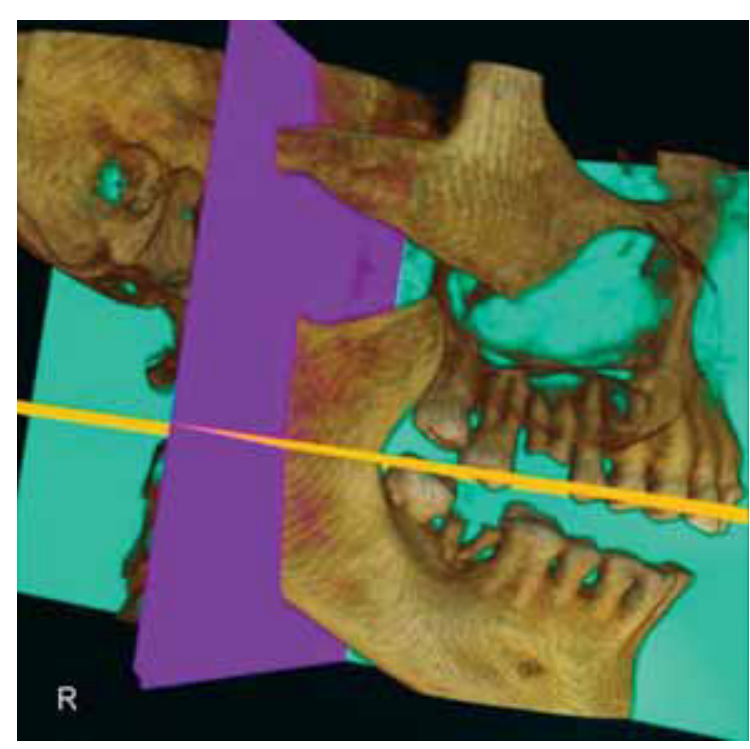

Fig. 4: 3D reconstruction image of the right-sided lesion
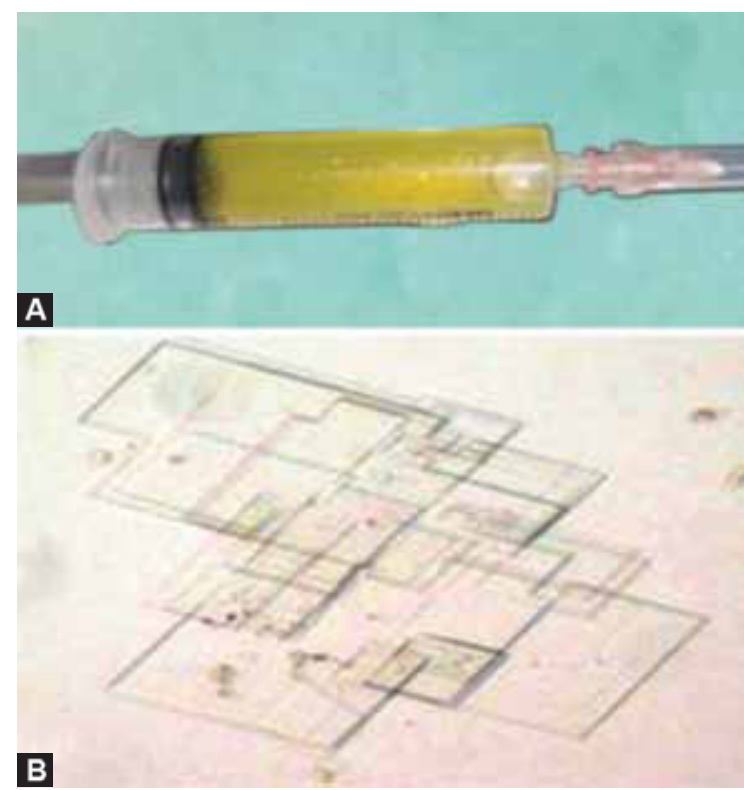

Figs 5A and B: (A) Aspiration fluid showed shimmering crystals,

(B) smear of the fluid showed numerous cholesterol crystals

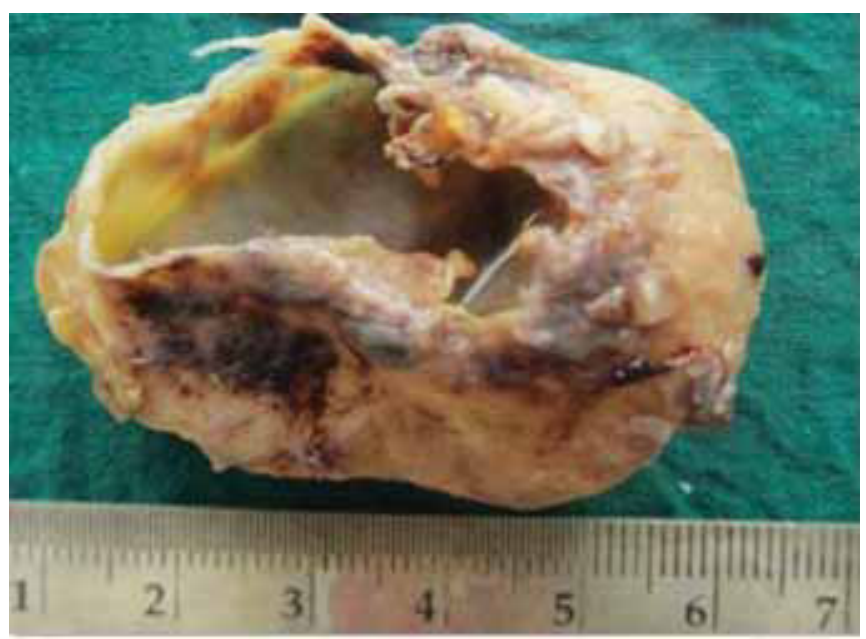

Fig. 6: Macroscopic image showing large cystic lesion 

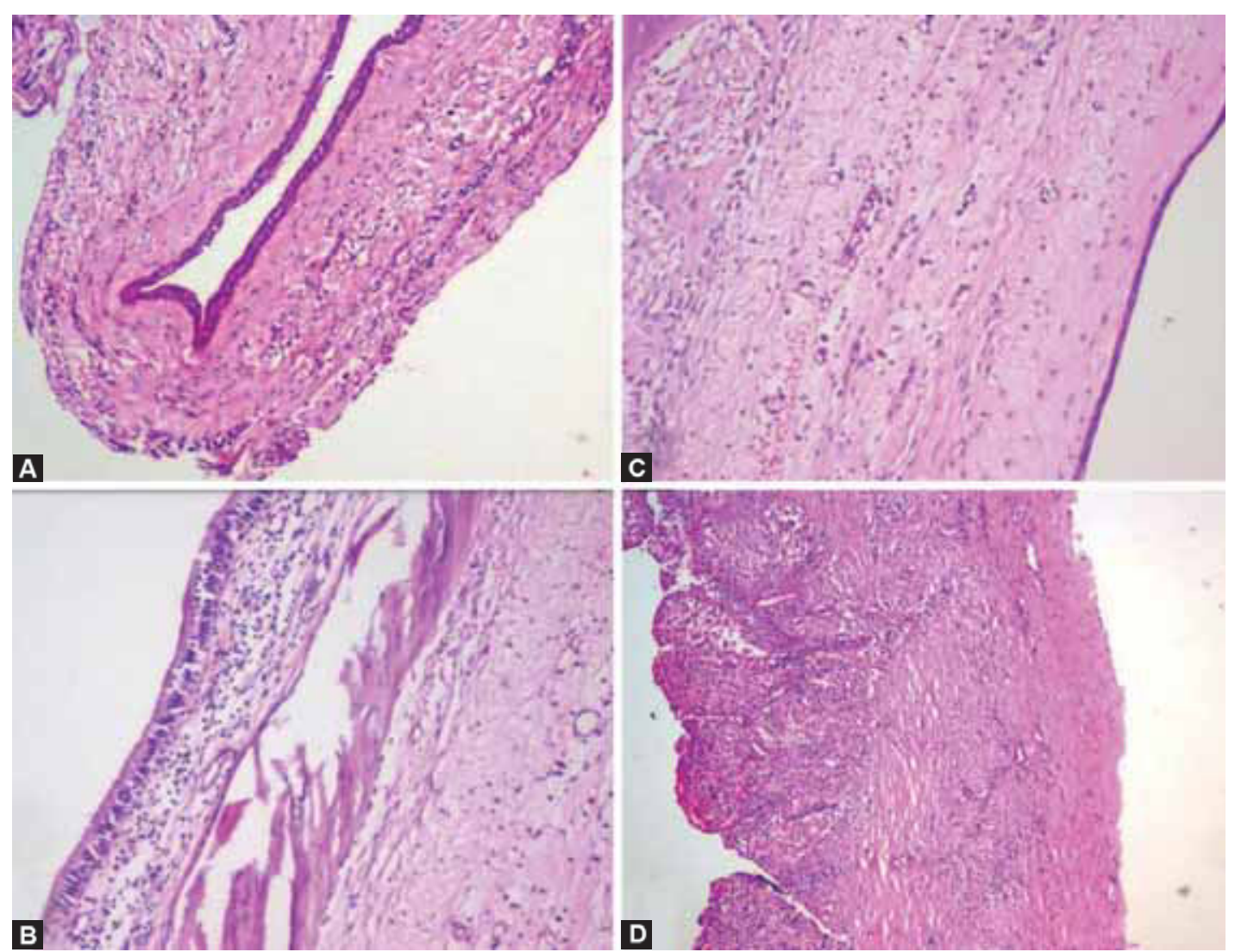

Figs 7A to D: (A) 100x; H \& E stained section showing cystic lumen lined by atrophic stratified squamous nonkeratinizing epithelium, (B) 100x; H \& E stained section showing pseudostratified ciliated epithelium, (C) 100x; H \& E stained section showing bland connective tissue capsule, (D) 200x; H \& E stained section showing area of hyperplastic spongiotic nonkeratinized stratified squamous epithelium exhibiting arcading pattern

dense inflammatory infiltrate in the underlying connective tissue. The connective tissue capsule showed mature collagen fibers with minimal cellularity which included fibroblasts, fibrocytes and inflammatory cells (Figs 7A to D). Components like cholesterol crystals which lead to inflammation were almost completely expelled into the cystic lumen. Few blood vessels and areas of hyalinization were evident.

The lining epithelium was similar to that of a dentigerous cyst, but since there was no associated impacted tooth or history of extraction with the underlying connective tissue showing mature collagen fibers, a diagnosis of infected dentigerous cyst was ruled out. It also lacked any similarity with odontogenic keratocystic lining.

As the patient had a 100-year history of recurrent pus discharge through right sinus tract irt first premolar, multiple carious teeth and PDL widening in relation to 16 , a diagnosis of radicular cyst was considered. Due to the history of multiple recurrences, the cyst would have matured over time and acquired a quiescent epithelial lining with a bland, fibrotic connective tissue wall. Considering these features, we came to a final diagnosis of quiescent radicular cyst.

\section{DISCUSSION}

The radicular cyst results from proliferation of epithelial remnants at the apex of a nonvital necrotic tooth in response to low intensity chronic stimulus triggered by bacterial endotoxins as well as their products and signals such as inflammatory mediators, proinflammatory cytokines, and growth factors released by innate and adaptive immune cells during apical periodontitis. The aggression is mainly confined to the periapical region. ${ }^{1,2,7}$

Various studies suggest that endotoxins have a major initiating role in the pathogenesis of radicular cysts and have a direct effect on epithelial proliferation. The major cytokines identified, interleukin (IL)-1 and IL-6, have a direct effect on epithelial proliferation. Endotoxins initiate an inflammatory response resulting in production of cytokines with proinflammatory and bone-resorbing activities. ${ }^{2}$

Factors such as the amount and type of cytokines; antigenic nature, adequate expression of receptors, costimulatory molecules, type of antigen-presenting cells and genetic constitution of the host; modulate the progression and severity of inflammatory processes as well as influence the type of host response. There are two types of effector responses in the body namely T-helper 1 (Th1) or T-helper 2 (Th2) whose responses are regulated by regulatory T cells ( $\mathrm{T}_{\text {reg }}$ cells). The mechanism of action of $\mathrm{T}_{\text {reg }}$ cells involves direct suppression of the activation of $\mathrm{T}$ and $\mathrm{B}$ lymphocytes and natural killer (NK) cells. ${ }^{8}$ 
In early cysts, the epithelial lining may be proliferative (6-20 cell layer thick) and show arcading with an intense associated inflammatory process. According to studies, it is suggested that presence of less effective regulatory environment ( $\mathrm{T}_{\text {reg }}$ cells) in RCs, associated with high levels of inflammatory mediators as reported in the literature, contribute to greater growth potential of the RCs at this stage. ${ }^{8}$ In RCs, the degree of inflammation might be associated with the proliferative activity of their epithelial cells; ${ }^{4}$ but, as the cyst enlarges, the site distant from the apex, has less antigenic stimulus and hence, less inflammatory cells in the connective tissue wall which allows the lining to become quiescent and fairly regular with certain degree of differentiation to resemble a simple stratified squamous epithelium. Adjacent to the apex, where the cyst is constantly exposed to the infected root canal, inflammation may persist, leading to hyperplastic epithelium. ${ }^{2}$

Our case correlates with this feature as it was a fairly large cystic lesion with quiescent epithelium and organized, less inflamed fibrous connective tissue wall at a site away from the periapical region of 16 . At this site the proliferation of the epithelium is probably limited by immunosuppressive responses. ${ }^{8}$ At the site close to the apex of the tooth where the antigenic stimulus still persists the epithelium showed proliferation with arcading pattern.

Epithelial proliferation continues as long as there is an inflammatory stimulus which contributes to enlargement of the cyst. When the stimulus to epithelial proliferation is reduced or ceased, cell proliferation probably continues, but at a relatively low level. Further an increase in the size of the cyst eventually leads to thinning of the epithelial lining. ${ }^{2}$

In addition, foreign body-type materials like cholesterol crystals, endodontic filling materials evokes an inflammatory reaction in the $\mathrm{CT}$ wall. Our case showed less of cholesterol crystals in the wall, they were mostly present in the cystic lumen which was laden heavily with the cholesterol crystals as seen in the aspiration fluid. This feature also suggests a better host response which was capable of eliminating all the crystals into the cystic lumen and thus lessening the inflammatory process. ${ }^{4}$

In our case, various features like a 10-year long history of persistence of infection with periodic exacerbation and regression, draining sinus potentiated by gravity as the lesion involved maxilla, and probably a better host response might have reduced the severity of the inflammatory response allowing for thinning of the epithelial lining giving it a quiescent nature.

\section{CONCLUSION}

Radicular cysts are inflammatory cysts with thick proliferative epithelium. It is rare to find this cyst to be almost completely lined by atrophic epithelium which suggests quiescent nature of the cyst with fairly regular epithelial lining. Such cysts widen the differential diagnosis and present a diagnostic dilemma due their similarity other cysts such as dentigerous cysts.

\section{ACKNOWLEDGMENTS}

It gives us immense pleasure in acknowledging the Staff, Department of Oral Pathology, Krishnadevaraya College of Dental Sciences, for their constant support and timely guidance.

\section{REFERENCES}

1. Holla VA, Chatra L, Shenai P, Rao PK, Veena KM, Prabhu RV. Bilateral inflammatory cysts of the jaw: report of an unusual case. Imag Sci Dent 2012;42:105-109.

2. Shear M, Speight PM. Cysts of the oral and maxillofacial regions. 4th ed. Victoria-Australia, Oxford: Blackwell; 2007. p. 123-141.

3. Amos MJ, Dalghous A, Alkhabuli J, Mizen KD. Massive maxillary radicular cyst presenting as facial fracture and abscess, a case report. Libyan J Med 2007;2:211-213.

4. Santos LC, Vilas Bôas DS, Oliveira GQ, Ramos EA, Gurgel CA, dos Santos JN. Histopathological study of radicular cysts diagnosed in a Brazilian population. Braz Dent J 2011;22(6): 449-454.

5. Kafas P, Kalfas S, Upile T, Jerjes W. Uncommon synchronous histopathological features of a radicular cyst: a case report. Cases J 2009;2:9067.

6. Pekiner FN, Borahan O, Uğurlu F, Horasan S, Şener BC, Olgaç V. Clinical and radiological features of a large radicular cyst involving the entire maxillary sinus. J Marmara Univ Ins Health Sci 2012;2(1):31-36.

7. Pitak-Arnnop P, Dhanuthai K, Hemprich A, Pausch NC. Huge radicular cyst of the maxilla: some clinicopathological considerations. Otolaryngol Head Neck Surg 2010;143:853-854.

8. Peixoto RF, Pereira Jdos S, Nonaka CF, Silveira EJ, Miguel MC. Immunohistochemical analysis of FoxP3+ cells in periapical granulomas and radicular cysts. Arch Oral Biol 2012 Sep;57(9):1159-1164.

\section{ABOUT THE AUTHORS}

\section{Reshma (Corresponding Author)}

Senior Lecturer, Department of Oral and Maxillofacial Pathology Krishnadevaraya College of Dental Sciences, Bengaluru, Karnataka India, Phone: 9916707538, e-mail: reshmav132@gmail.com

\section{Radhika M Bavle}

Professor and Head, Department of Oral and Maxillofacial Pathology Krishnadevaraya College of Dental Sciences, Bengaluru, Karnataka, India

\section{BK Varsha}

Senior Lecturer, Department of Oral and Maxillofacial Pathology Krishnadevaraya College of Dental Sciences, Bengaluru, Karnataka, India

\section{BM Kavya}

Senior Lecturer, Department of Oral and Maxillofacial Pathology Krishnadevaraya College of Dental Sciences, Bengaluru, Karnataka India 